\title{
Reaction of monosaccharides with 2-pyridylcarboxamidrazone and determination of the nature of products
}

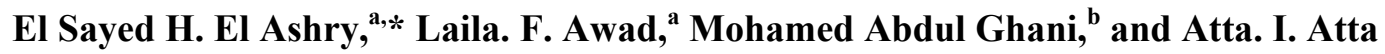 \\ ${ }^{a}$ Chemistry Department, Faculty of Science, Alexandria University, Alexandria, Egypt \\ ${ }^{b}$ Chemistry Department, Faculty of Science, Beirut Arab University, Beirut, Lebanon \\ E-mail: eelashry60@hotmail.com,eelashry60@link.net
}

(received 14 Jul 05; accepted 22 Sep 05; published on the web 07 Oct 05)

\begin{abstract}
Reaction of 2-pyridylcarboxamidrazone with D-arabinose, D-xylose, D-galactose, D-glucose and D-mannose afforded the corresponding hydrazones in either the acyclic or cyclic structures depending on the nature of the sugar. Assignment of the sugar hydrazones structures were based on 1D and 2D NMR experiments. Vicinal coupling constants were used to deduce the favored conformations.
\end{abstract}

Keywords: Carbohydrates, sugar hydrazones, amidrazones, seco-nucleosides

\section{Introduction}

Amidrazones are important precursors for many useful compounds with industrial and medicinal applications. ${ }^{1} \quad$ 2-Pyridylcarboxamidrazone and its heterocyclic derivatives showed antimicrobacterial activity $^{2}$ and anticancer activity. ${ }^{3}$ The phenylalanine derivative (S)-3-(4amidrazonophenyl)- $N$-cyclopentyl- $N$-methyl-2-(naphthalene-2-sulfonyl)propionamide (LB30057) was identified as a potent selective and orally active thrombin inhibitor; several derivatives have also significant enhancing potency as thrombin inhibitors. ${ }^{4}$ Carbohydrates have attracted much attention as starting material in organic synthesis. ${ }^{5}$ Their role in the synthesis of naturally occurring nitrogen heterocycles ${ }^{6}$ has been investigated in various laboratories.

Much work has been published from our laboratory on the synthesis and reactivity of hydrazones ${ }^{7}$ derived from carbohydrates for the synthesis of heterocycles ${ }^{6}$ and acyclic (seco)nucleosides. ${ }^{8}$ The reaction of sugars with benzamidrazone (A) has led to an interesting entry towards nucleoside analogues. ${ }^{9}$ Moreover, their reaction with 2-pyridylhydrazine (B) has been recently investigated. ${ }^{10}$ D-Glucose 2-pyridylhydrazone was isolated during a study on sequential removal of monosaccharides from the reducing end of oligosaccharides. ${ }^{11}$ Consequently, it became interesting to investigate the reactions of the close analogue, 2-pyridylcarboxamidrazone (1), which has both features. The incorporation of alditolyl moieties on these pyridylamidrazones 
may lead to an interesting improvement in their biological activity and/or enhancement of their bioavailability as a consequence of the hydrophilic nature of the alditolyl residue that may aid in their transportation into biological systems.

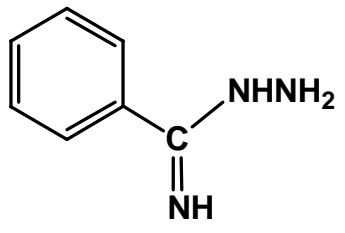

A

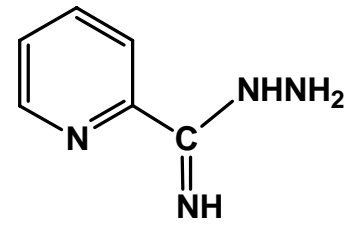

1

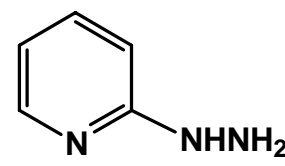

B

\section{Figure 1}

\section{Results and Discussion}

Condensation of an equimolar amount of 2-pyridylcarboxamidrazone (1) with aldoses was carried out in refluxing ethanol to give the respective hydrazone derivatives 5, 6, 10, 11 and 12 accompanied with a yellow crystalline by-product, which was separated by fractional crystallization and identified from its spectral data; FABMS showed a molecular ion peak at m/z 240 as the dihydrazidine $2 .{ }^{12}$ Moreover, the dihydrazidine $\mathbf{2}$ was formed when the amidrazone $\mathbf{1}$ was heated in a solution of ethanol and in the presence of oxygen.

Sugar hydrazones can exist in solution as acyclic Schiff's base or as cyclic glycosylhydrazine ${ }^{10,13,14}$ and are often present as equilibrium mixtures that are strongly dependent on the $\mathrm{pH}$ of the solution as well as on the nature of the sugar and the basicity of the hydrazine derivative. The characterization and assignment of the acyclic-cyclic nature of the newly prepared sugar hydrazones were investigated based on the study of their ${ }^{1} \mathrm{H}$ and ${ }^{13} \mathrm{C}$ NMR spectra, while ${ }^{1} \mathrm{H}-{ }^{1} \mathrm{H}$ DQFCOSY and ${ }^{1} \mathrm{H}-{ }^{13} \mathrm{C}$ HMQC NMR experiments helped in the assignment of both proton and carbon signals.

Examination of ${ }^{1} \mathrm{H}$ and ${ }^{13} \mathrm{C}$ NMR spectra of the D-arabinose derivative $\mathbf{5}$ in a solution of DMSO- $\mathrm{d}_{6}$ and $\mathrm{D}_{2} \mathrm{O}$ showed that it existed exclusively in the acyclic structure; based on the presence of a doublet corresponding to $\mathrm{H}-1^{\prime}$ at the downfield region at $\delta 7.77$ that correlated with $\mathrm{C}-1{ }^{\prime}$ at $\delta_{\mathrm{C}} 159.7$. The presence of only four signals corresponding to the sugar carbons at $\delta_{\mathrm{C}} 63.8,70.8,71.5,73.1\left(\mathrm{C}-5^{\prime}, 2^{\prime}\right.$, 4 ' and $3^{\prime}$ ) was also in agreement of such acyclic structure.

Conversely, D-xylose (4) afforded a product whose NMR spectral data (DMSO- $\left.\mathrm{d}_{6}+\mathrm{D}_{2} \mathrm{O}\right)$ indicated the presence of more than one component with the most prominent being the two anomers $N^{2}-(\alpha, \beta-\mathrm{D}-$ xylopyranosyl)- $N^{1}$-2-pyridylcarboxamidrazone ( $6 \mathbf{b} \alpha$ and $\left.\mathbf{6 b} \beta\right)$, present in a 1:1.6 ratio. Identification of both anomers was performed by assignment of the signals at the downfield region corresponding to $H-1^{\prime}-\alpha\left(\delta\right.$ 4.86) and H-1'- $\beta(\delta 4.23)$ which appeared as doublets with vicinal coupling constants, $\mathrm{J}_{1^{\prime}, 2^{\prime}}=$ $3.5 \mathrm{~Hz}$ for the $\alpha$ anomer compatible with a cis relationship with $\mathrm{H}-2$ ', whereas that of the $\beta$-anomer, $\mathrm{J}_{1^{\prime}, 2^{\prime}}=7.7 \mathrm{~Hz}$, agreed with a trans relationship for H-1', H-2'. A HMQC experiment correlated H-1' $\alpha$ 
and $\mathrm{H}-1$ ' $\beta$ with the carbons resonating at $\delta_{\mathrm{C}} 91.3$ and 96.6 , respectively, in agreement with the expected cyclic structure. The presence of the cyclic structure in the pyranose form rather than the furanose form was confirmed by the signal of C-4' which appeared at a lower frequency region at $\delta_{\mathrm{C}}$ 69.0 and 68.6 for $\mathbf{6 b} \alpha$ and $\mathbf{6 b} \beta$, respectively, in agreement with those established for pyranosides; ${ }^{15}$ expected values for furanosides were $\delta_{\mathrm{C}} 77.4$ and $72.7 .{ }^{16}$ The assignment of the signals at $\delta_{\mathrm{C}} 60.3$ and 64.5 for C-5' of both anomers $6 \mathrm{~b} \alpha$ and $6 \mathrm{~b} \beta$, respectively, was based on DEPT- $135^{\circ}$ experiments. These anomers were the two forms existing in solution and no signals corresponding to the acyclic structure 6a were observed, although $6 \mathbf{b} \alpha$ and $6 \mathrm{~b} \beta$ need to be formed via the open chain hydrazone $6 \mathbf{a}$. However, it seems that the cyclic structures are readily formed and consequently $\mathbf{6 a}$ can not be detected.

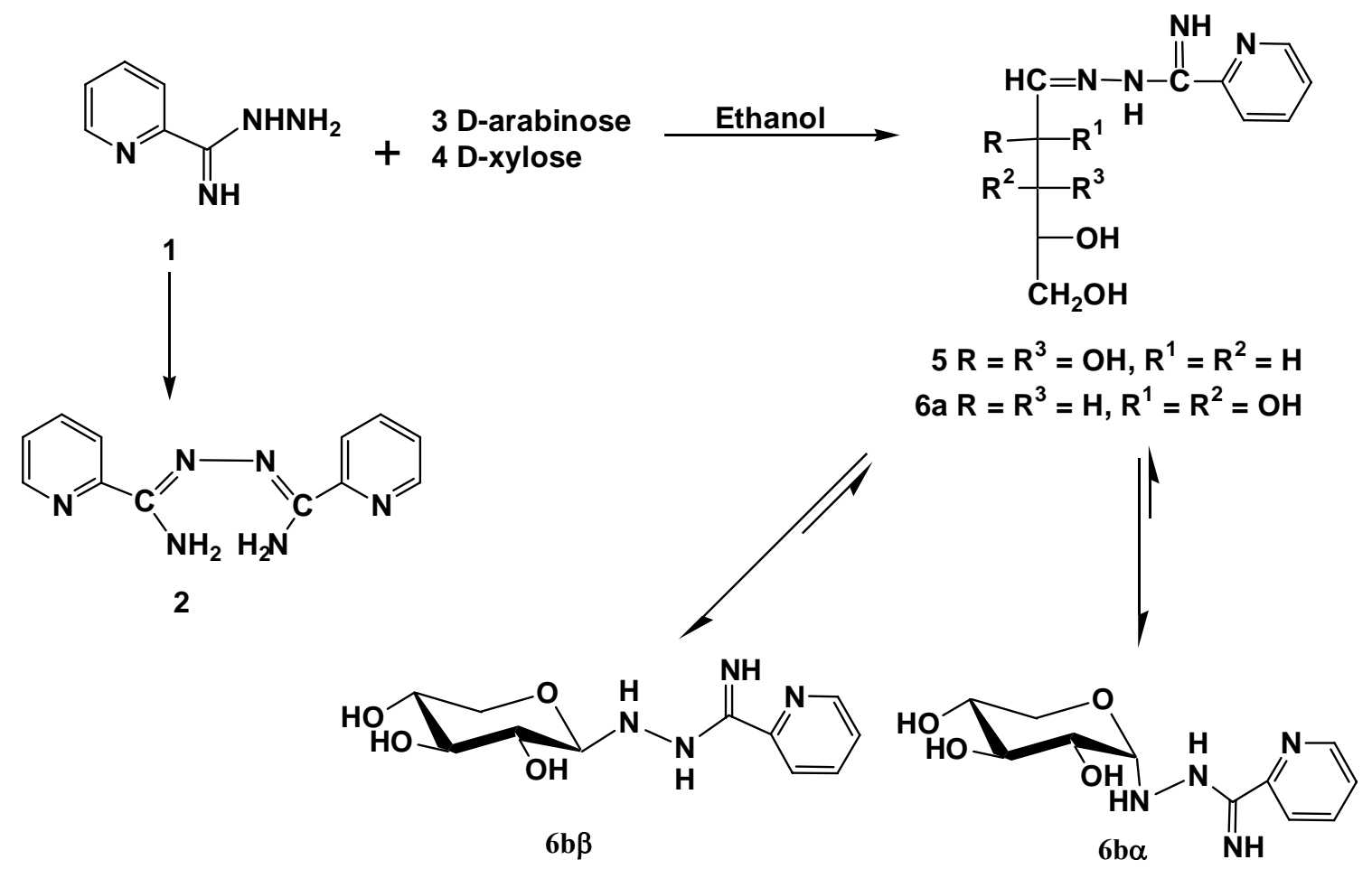

\section{Scheme 1}

Spectral analysis of the product from D-galactose (7) showed that it existed as a mixture of the acyclic 10a and cyclic 10b structure, in a ratio of 4:1, respectively, in which the acyclic structure was the main one. The presence of a doublet corresponding to $\mathrm{H}-1^{\prime}$ in the downfield region at $\delta 7.80$ that correlated with $\mathrm{C}-1^{\prime}$ at $\delta_{\mathrm{C}} 159.9$ confirmed the presence of the acyclic structure. The pyranosyl ring structure in 10b was assigned based on the signal corresponding to C-5' that resonated at $\delta_{\mathrm{C}} 76.4$. The presence of only one signal corresponding to $\mathrm{H}-1$ ' at $\delta 4.20$, correlated with $\mathrm{C}-1^{\prime}$ at $\delta \mathrm{c} 91.7$, indicated the presence of only a single anomer $\mathbf{1 0 b}$. The assignment of the anomeric configuration of $\mathbf{1 0 b}$ as $\beta$ was based on the value of $J_{1^{\prime}, 2^{\prime}}=8.9 \mathrm{~Hz}$, confirming a trans relationship with $\mathrm{H}-2^{\prime}$. 
The D-mannose derivative $\mathbf{1 1}$ also existed in the acyclic and cyclic structures in a ratio of 4:1, respectively. Thus, H-1' in the acyclic structure 11a appeared as a doublet at $\delta 7.84$, correlated with C$1^{\prime}$ at $\delta_{\mathrm{C}} 159.9$, while the cyclic pyranosyl structure $11 \mathbf{b}$ was based on the assignment of C-5' at $\delta_{\mathrm{C}} 78.7$. Its $\mathrm{H}-1^{\prime}$ was observed at $\delta 4.48$, correlated with $\mathrm{C}-1^{\prime}$ at $\delta_{\mathrm{C}} 88.0$, and showed a coupling constant $\mathrm{J}_{1^{\prime}, 2^{\prime}}=$ $8.6 \mathrm{~Hz}$ which was not fully decisive for assigning the $\alpha$ or $\beta$-anomeric configuration for $\mathbf{1 1 b}$.

The adduct from D-glucose (9) and 1 was found to be the acyclic structure 12a, the $\alpha, \beta$-pyranosyl 12b and the $\alpha, \beta$-furanosyl 12c ring structure, in which the cyclic structure was the main one as deduced from their ${ }^{1} \mathrm{H}$ and ${ }^{13} \mathrm{C}$ NMR spectra in DMSO- $\mathrm{d}_{6}$ and $\mathrm{D}_{2} \mathrm{O}$. At the higher frequency region the azomethine carbon $\left(\mathrm{C}-1^{\prime}=\mathrm{N}\right)$ of the acyclic form 12a was observed at $\delta_{\mathrm{C}} 162.9$. The presence of more than one cyclic structure was deduced from the presence of four anomeric signals; assigned at $\delta_{\mathrm{C}} 90.9$, 91.4, 92.6 (lower intensities) and 97.2 (higher intensity). In addition, the DEPT- $135^{\circ}$ experiment showed the presence of five negative signals at $\delta_{\mathrm{C}} 61.4,61.5,61.7,63.1,63.8$ corresponding to C-6' in agreement with the presence of five species. Complete assignment of either sugar protons or carbons was not possible due to the overlap of their signals. The doublet of the $\beta$-anomeric proton of the pyranosyl isomer 12b was assigned at $\delta 4.28$ with $\mathrm{J}_{1^{\prime}, 2^{\prime}}=7.6 \mathrm{~Hz}$, correlating with the triplet of $\mathrm{H}-2^{\prime}$ at $\delta$ 2.89 that consequently correlated with $\mathrm{H}-3^{\prime}$ at $\delta 3.01$. The pyranosyl ring structure was confirmed from the downfield assignment of $\mathrm{C}-5^{\prime}$ at $\delta_{\mathrm{C}} 78.1 .{ }^{1} \mathrm{H}^{13} \mathrm{C}-\mathrm{HMQC}$ experiment showed that the signals corresponding to C-1', C-2', C-3' and C-4' resonated at $\delta_{C} 97.2,75.1,70.6$ and 77.1, respectively. The anomeric $\alpha$-proton was observed at $\delta 4.90$ which correlated with the doublet of doublets of $\mathrm{H}-2^{\prime} \alpha$ at $\delta$ 3.14 with $\mathrm{J}_{1^{\prime}, 2^{\prime}}=3.6 \mathrm{~Hz}$, whereas their corresponding carbons resonated at $\delta_{\mathrm{C}} 92.6$ and 70.9 , respectively.

Signals corresponding to protons and carbons of the pyridyl moiety for all products were similar and were assigned based on $1 \mathrm{D}$ and $2 \mathrm{D}$ NMR experiments. The ${ }^{1} \mathrm{H}$ NMR spectra of all products typically showed H-6 as the most downfield shift at $\delta_{\mathrm{H}} 8.59$, as a doublet correlating to $\mathrm{H}-5^{\prime}\left(\delta_{\mathrm{H}} 7.49\right)$, appearing as a dd, which in turn was correlating to $\mathrm{H}-4\left(\delta_{\mathrm{H}} 7.86\right)$, appearing as a ddd which correlated to $\mathrm{H}-3\left(\delta_{\mathrm{H}} 8.08\right)$ that appeared as a doublet. Signals for the respective carbons were assigned based on ${ }^{13} \mathrm{C}$, DEPT-135 ${ }^{\circ}$ and ${ }^{1} \mathrm{HMQC}$ experiments; observed at $\delta_{\mathrm{C}} 149.2$ (C-6), 126.2 (C-5), $137.8(\mathrm{C}-4), 121.6$ (C-3) while $\mathrm{C}-2$ showed as quaternary carbon in the region $\delta_{\mathrm{C}} 150.5 \mathrm{ppm}$. The $\mathrm{C}=\mathrm{NH}$ amidrazone carbon was assigned at $\delta_{\mathrm{C}} 156.7$. 


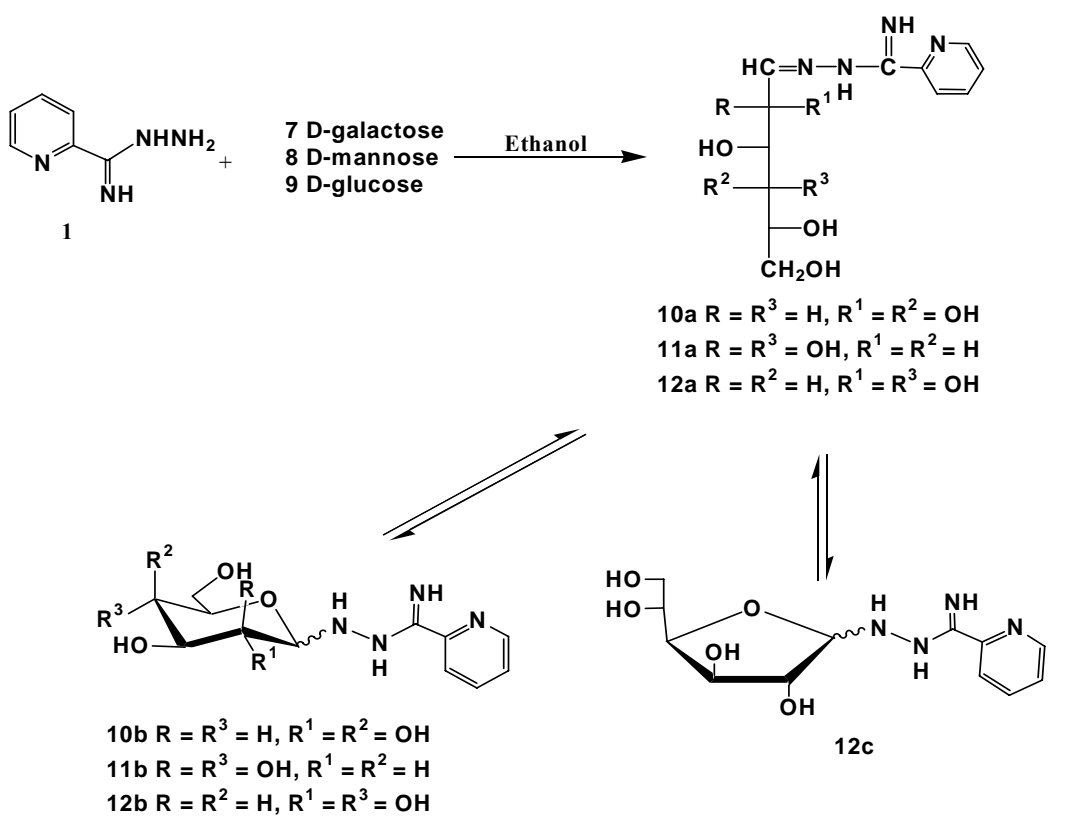

\section{Scheme 2}

Reaction of D-xylose and D-glucose with amidrazone 1, gave products in which the pyranose ring structure was predominant whereas the acyclic structure was found to be more favorable for the products from D-arabinose, D-mannose and D-galactose. The carbohydrate structure and the nature of the amidrazone determines the ratio of the acyclic and/or cyclic compounds in solution. ${ }^{9}$

\section{Experimental Section}

General Procedures. Melting points were determined on a Mel-temp apparatus and are uncorrected. Mass spectra were recorded using Electron Impact (EI) on a Finnian MAT 312 spectrometer and Fast-Atom bombardment (FAB) on a Kratos MS 50 spectrometer. ${ }^{1} \mathrm{H}$ NMR and ${ }^{13} \mathrm{C}$ NMR spectra were recorded on a Bruker DRX $600 \mathrm{MHz}$ or a Bruker Avance $300 \mathrm{MHz}$ spectrometer. The chemical shifts are expressed on the $\delta$-scale using $\mathrm{Me}_{4} \mathrm{Si}$ as a standard, and coupling-constant values are given in Hz. The assignments of ${ }^{1} \mathrm{H}$ NMR spectra were based on chemical-shift correlation DQFCOSY spectra, while the assignment of ${ }^{13} \mathrm{C}$ NMR spectra were based on heteronuclear multiple quantum coherence (HMQC) experiments. TLC was preformed on Merck Silica Gel 60F254 with detection by charring in sulfuric acid and by UV light. Microanalyses were preformed in the Microanalysis Unit at the Faculty of Science, Cairo University.

\section{General procedure for reaction of 2-pyridylcarboxamidrazone with aldoses}

A mixture of the aldose $(10 \mathrm{mmol})$ and 2-pyridylcarboxamidrazone 1 (10 $\mathrm{mmol})$ in ethanol $(50 \mathrm{ml})$ was heated under reflux for 2-5 hours. The reaction mixture was then concentrated and left to cool. Fractional crystallization of the product gave the amidrazones and dihydrazidine. 
$N^{2}$-(D-Arabinose)- $\boldsymbol{N}^{\mathbf{1}}$-2-pyridylcarboxamidrazone (5). Pale yellow crystals (78 \%), m.p. 168$170{ }^{\circ} \mathrm{C},{ }^{1} \mathrm{H}$ NMR $\left(600 \mathrm{MHz}, \mathrm{DMSO}-\mathrm{d}_{6}+\mathrm{D}_{2} \mathrm{O}\right) \delta(\mathrm{ppm}) 3.43\left(\mathrm{dd}, 1 \mathrm{H}, \mathrm{H}-5^{\prime}, J_{4^{\prime}, 5^{\prime}}=5.9 \mathrm{~Hz}, J_{5^{\prime}, 5^{\prime \prime}}\right.$ $=11.2 \mathrm{~Hz}), 3.50\left(\mathrm{dd}, 1 \mathrm{H}, \mathrm{H}-3^{\prime}, J_{2^{\prime}, 3^{\prime}}=2.4 \mathrm{~Hz}, J_{3^{\prime}, 4^{\prime}}=8.4 \mathrm{~Hz}\right), 3.54-3.57$ (m, $\left.1 \mathrm{H}, \mathrm{H}-4^{\prime}\right), 3.62$ (dd, $\left.1 \mathrm{H}, \mathrm{H}-5^{\prime \prime}, J_{4^{\prime}, 5^{\prime \prime}}=3.3 \mathrm{~Hz}, J_{5^{\prime}, 5^{\prime \prime}}=11.2 \mathrm{~Hz}\right), 4.46\left(\mathrm{dd}, 1 \mathrm{H}, \mathrm{H}-2^{\prime}, J_{1^{\prime}, 2^{\prime}}=4.2 \mathrm{~Hz}, J_{2^{\prime}, 3^{\prime}}=2.4 \mathrm{~Hz}\right), 7.49$ $\left(\mathrm{dd}, 1 \mathrm{H}, \mathrm{H}-5, J_{5,6}=4.9 \mathrm{~Hz}, J_{4,5}=7.6 \mathrm{~Hz}\right), 7.77$ (d, $\left.1 \mathrm{H}, \mathrm{H}-1^{\prime}, J_{1^{\prime}, 2^{\prime}}=4.2 \mathrm{~Hz}\right), 7.86$ (ddd, $1 \mathrm{H}, \mathrm{H}-4$, $\left.J_{4,3}=7.8 \mathrm{~Hz}, J_{4,5}=7.6 \mathrm{~Hz}, J_{4,6}=1.4 \mathrm{~Hz}\right), 8.08\left(\mathrm{~d}, 1 \mathrm{H}, \mathrm{H}-3, J_{3,4}=7.9 \mathrm{~Hz}\right), 8.59\left(\mathrm{~d}, 1 \mathrm{H}, \mathrm{H}-6, J_{5,6}\right.$ $=4.9 \mathrm{~Hz}) .{ }^{13} \mathrm{C}$ NMR $\left(150.8 \mathrm{MHz}, \mathrm{DMSO}-\mathrm{d}_{6}+\mathrm{D}_{2} \mathrm{O}\right) \delta(\mathrm{ppm}) 63.8\left(\mathrm{C}-5^{\prime}\right), 70.8\left(\mathrm{C}-2^{\prime}\right), 73.1\left(\mathrm{C}-3^{\prime}\right)$, 71.5 (C-4'), 121.6 (C-3), 126.2 (C-5), 137.8 (C-4), 149.2 (C-6), 150.5 (C-2), 156.7 (NC=N), $159.7\left(\mathrm{C}-1^{\prime}\right)$; FABMS $\mathrm{m} / \mathrm{z}=269\left(\mathrm{MH}^{+\cdot}\right)$. Analylsis for $\mathrm{C}_{11} \mathrm{H}_{16} \mathrm{~N}_{4} \mathrm{O}_{4}(268.27)$, calcd: $\mathrm{C}, 49.25 ; \mathrm{H}$, $6.01 ;$ N, 20.88; Found: C, 49.12; H, 6.05; N, 20.71\%.

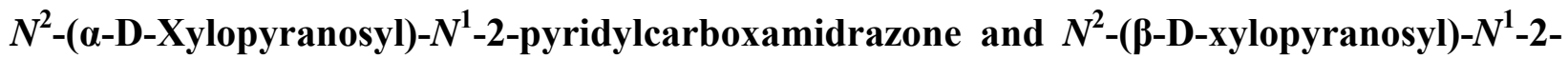
pyridylcarboxamidrazone (6ba and $6 \mathbf{b} \beta)$. Hygroscopic glassy product $(78 \%), \alpha: \beta(2: 3),{ }^{1} \mathrm{H}$ NMR (300 MHz, DMSO-d $\left.{ }_{6}+\mathrm{D}_{2} \mathrm{O}\right)$ for $\alpha$-anomer $\mathbf{6 b} \alpha \delta(\mathrm{ppm}) 3.16\left(\mathrm{~d}, 0.4 \mathrm{H}, \mathrm{H}-2^{\prime}, J_{1^{\prime}, 2^{\prime}}=3.5\right.$ Hz), 3.27-3.31 (m, 0.4 H, Hz, H-4'), 3.38 (dd, 0.4 H, H-3', $J_{3^{\prime}, 4^{\prime}}=7.6 \mathrm{~Hz}$ ), 3.35-3.42 (m, 0.8 H, H-5',5"), 4.86 (d, 0.4 H, H-1'- $\alpha, J_{1^{\prime}, 2^{\prime}}=3.5 \mathrm{~Hz}$ ). ${ }^{13} \mathrm{C}$ NMR (DMSO-d 6 ) $\delta(\mathrm{ppm}) 60.3\left(\mathrm{C}-5{ }^{\prime}\right), 69.0$ (C-4'), $71.1\left(\mathrm{C}^{\prime} 2^{\prime}\right), 71.9\left(\mathrm{C}-3^{\prime}\right), 91.3\left(\mathrm{C}-1^{\prime}-\alpha\right)$, FABMS $m / z=268\left(\mathrm{M}^{+\cdot}\right)$.

${ }^{1} \mathrm{H}$ NMR ( DMSO-d $6_{6}+\mathrm{D}_{2} \mathrm{O}$ ) for $\beta$-anomer $6 \mathbf{b} \beta \delta(\mathrm{ppm}) 2.89\left(\mathrm{t}, 0.6 \mathrm{H}, \mathrm{H}-2^{\prime}, J_{1^{\prime}, 2}{ }^{\prime}=J_{2^{\prime}, 3^{\prime}}=8.9\right.$ $\mathrm{Hz}$ ), 3.09 (dd, 0.6 H, H-5', $\left.J_{5^{\prime}, 4^{\prime}}=8.9 \mathrm{~Hz}, J_{5^{\prime}, 5^{\prime \prime}}=11.3 \mathrm{~Hz}\right), 3.12$ ( m, 0.6 H, H-3'), 3.30 (m, 0.6 H, H-4'), 3.65 (dd, 0.6 H, H-5", $\left.J_{4^{\prime}, 5^{\prime \prime}}=5.4 \mathrm{~Hz}, J_{5^{\prime}, 5^{\prime \prime}}=11.3 \mathrm{~Hz}\right), 4.23$ (d, 0.6 H, H-1'- $\beta, J_{1^{\prime}, 2^{\prime}}=7.7$ Hz). ${ }^{13} \mathrm{C}$ NMR (DMSO-d 6 ) $\delta(\mathrm{ppm}) 64.5$ (C-5'), 68.6 (C-4'), 73.5 (C-2'), 75.5 (C-3'), 96.6 (C-1'$\beta)$.

$N^{2}$-(D-Galactose)- $N^{1}$-2-pyridylcarboxamidrazone and $\quad N^{2}$-( $\beta$-D-galactopyranosyl)- $N^{1}-2-$ pyridylcarboxamidrazone (10a,b). Pale yellow crystals $(65 \%)$, m.p. 124-126 ${ }^{\circ} \mathrm{C},{ }^{1} \mathrm{H}$ NMR $\left(600 \mathrm{MHz}, \mathrm{DMSO}-\mathrm{d}_{6}+\mathrm{D}_{2} \mathrm{O}\right)$ for 10a $\delta(\mathrm{ppm}) 3.38\left(\mathrm{dd}, 1.6 \mathrm{H}, \mathrm{H}-6^{\prime}, 6^{\prime \prime}, J_{6^{\prime}, 5^{\prime}}=5.2 \mathrm{~Hz}, J_{6^{\prime \prime}, 5^{\prime}}=6.6\right.$ $\left.\mathrm{Hz}, J_{6^{\prime}, 6^{\prime \prime}}=9.5 \mathrm{~Hz}\right), 3.56\left(\mathrm{~d}, 0.8 \mathrm{H}, \mathrm{H}-4^{\prime}, J_{4^{\prime}, 3^{\prime}}=9.2 \mathrm{~Hz}\right.$ ), 3.76 (under DMSO, H-3'), 4.49 (d, 0.8 $\left.\mathrm{H}, \mathrm{H}-2^{\prime}, J_{2^{\prime}, 3^{\prime}}=2.3 \mathrm{~Hz}\right), 7.49\left(\mathrm{dd}, 0.8 \mathrm{H}, \mathrm{H}-5^{\prime}, J_{5^{\prime}, 6^{\prime}}=5.2 \mathrm{~Hz}, J_{5^{\prime}, 4^{\prime}}=6.7 \mathrm{~Hz}\right), 7.80$ (d, $0.8 \mathrm{H}, \mathrm{H}-1^{\prime}$, $\left.J_{1^{\prime}, 2^{\prime}}=4.1 \mathrm{~Hz}\right), 7.86\left(\mathrm{ddd}, 0.8 \mathrm{H}, \mathrm{H}-4, J_{3,4}=7.9 \mathrm{~Hz}, J_{4,5}=1.30 \mathrm{~Hz}\right), 8.10\left(\mathrm{~d}, 0.8 \mathrm{H}, \mathrm{H}-3, J_{3,4}=7.9\right.$ $\mathrm{Hz}), 8.60$ (d, $\left.0.8 \mathrm{H}, \mathrm{H}-6, J_{5,6}=4.5 \mathrm{~Hz}\right)$.

${ }^{13} \mathrm{C}$ NMR (150.8 MHz, DMSO-d 6 ) $\delta(\mathrm{ppm}) 63.3\left(\mathrm{C}-6^{\prime}\right), 69.6\left(\mathrm{C}-4^{\prime}\right), 70.1\left(\mathrm{C}-5^{\prime}\right), 70.8\left(\mathrm{C}-2^{\prime}\right)$, 71.9 (C-3'), 121.7 (C-3), 125.9 (C-5), 137.5 (C-4), 148.9 (C-6), 150.5 (C-2), $156.4(\mathrm{NC}=\mathrm{N})$, $159.9\left(\mathrm{C}-1^{\prime}\right)$.

${ }^{1} \mathrm{H}$ NMR (600 MHz, DMSO-d $\left.6_{6}+\mathrm{D}_{2} \mathrm{O}\right)$ for $\mathbf{1 0 b} \delta(\mathrm{ppm}) 3.40\left(\mathrm{~d}, 0.2 \mathrm{H}, \mathrm{H}-3^{\prime}, J_{3^{\prime}, 4^{\prime}}=3.6 \mathrm{~Hz}\right)$, 3.41 (under DMSO, H-5'), 3.47 (dd, $0.4 \mathrm{H}, \mathrm{H}-6^{\prime}, 6^{\prime \prime}, J_{6^{\prime}, 5^{\prime}}=4.7 \mathrm{~Hz}, J_{6^{\prime \prime}, 5^{\prime \prime}}=6.3 \mathrm{~Hz}, J_{6^{\prime}, 6^{\prime \prime}}=11.8$ $\mathrm{Hz}$ ), 3.63 (dd, $0.2 \mathrm{H}, \mathrm{H}-2^{\prime}, J_{1^{\prime}, 2^{\prime}}=8.9 \mathrm{~Hz}, J_{2^{\prime}, 3^{\prime}}=8.2 \mathrm{~Hz}$ ), 3.76 (under DMSO, H-4'), 4.20 (d, 0.2 $\left.\mathrm{H}, \mathrm{H}-1^{\prime}, J_{1^{\prime}, 2^{\prime}}=8.9 \mathrm{~Hz}\right) .{ }^{13} \mathrm{C}$ NMR $\left(150.8 \mathrm{MHz}, \mathrm{DMSO}-\mathrm{d}_{6}\right) \delta(\mathrm{ppm}) 61.2\left(\mathrm{C}-6^{\prime}\right), 68.9\left(\mathrm{C}-4^{\prime}\right), 71.9$ (C-2'), 74.5 (C-3'), 76.4 (C-5'), 91.7 (C-1'); FABMS m/z = $299\left(\mathrm{MH}^{+\cdot}\right)$. Analysis for $\mathrm{C}_{12} \mathrm{H}_{18} \mathrm{~N}_{4} \mathrm{O}_{5}$ (298.30): Calcd: C, 48.32; H, 6.08; N, 18.78. Found: C, 48.11; H, 5.91; N, 18.59\%.

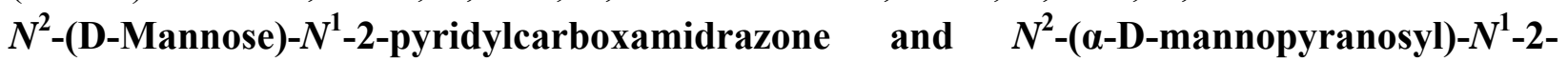
pyridylcarboxamidrazone (11a,b). Pale yellow crystals $(80 \%)$, m.p. $164-166{ }^{\circ} \mathrm{C},{ }^{1} \mathrm{H}$ NMR $(600$ $\left.\mathrm{MHz}, \mathrm{DMSO}-\mathrm{d}_{6}+\mathrm{D}_{2} \mathrm{O}\right)$ for $11 \mathrm{a} \delta(\mathrm{ppm}) 3.42\left(\mathrm{dd}, 0.8 \mathrm{H}, \mathrm{H}-6^{\prime}, J_{5^{\prime}, 6^{\prime}}=6.1 \mathrm{~Hz}, J_{6^{\prime}, 6^{\prime \prime}}=11.7 \mathrm{~Hz}\right)$, 
3.48-3.50 (m, $\left.0.8 \mathrm{H}, \mathrm{H}-5^{\prime}\right), 3.60$ (t, $\left.0.8 \mathrm{H}, \mathrm{H}-4^{\prime}, J_{4^{\prime}, 3^{\prime}}=J_{4^{\prime}, 5^{\prime}}=8.5 \mathrm{~Hz}\right), 3.63$ (dd, $0.8 \mathrm{H}, \mathrm{H}-6^{\prime \prime}, J_{5^{\prime}, 6^{\prime \prime}}$ $\left.=2.9 \mathrm{~Hz}, J_{6^{\prime}, 6^{\prime \prime}}=11.7 \mathrm{~Hz}\right), 3.69\left(\mathrm{t}, 0.8 \mathrm{H}, \mathrm{H}-3^{\prime}, J_{3^{\prime}, 4^{\prime}}=J_{2^{\prime}, 3^{\prime}}=8.5 \mathrm{~Hz}\right), 4.21\left(\mathrm{dd}, 0.8 \mathrm{H}, \mathrm{H}-2^{\prime}, J_{1^{\prime}, 2^{\prime}}=\right.$ $\left.4.8 \mathrm{~Hz}, J_{2^{\prime}, 3^{\prime}}=8.5 \mathrm{~Hz}\right), 7.49\left(\mathrm{dd}, 0.8 \mathrm{H}, \mathrm{H}-5, J_{5,6}=5.3 \mathrm{~Hz}, J_{4,5}=6.8 \mathrm{~Hz}\right), 7.84\left(\mathrm{~d}, 0.8 \mathrm{H}, \mathrm{H}-1^{\prime}\right.$, $\left.J_{1^{\prime}, 2^{\prime}}=4.9 \mathrm{~Hz}\right), 7.88\left(\mathrm{dd}, 0.8 \mathrm{H}, \mathrm{H}-4, J_{3,4}=7.9 \mathrm{~Hz}, J_{4,5}=6.8 \mathrm{~Hz}\right), 8.09\left(\mathrm{~d}, 0.8 \mathrm{H}, \mathrm{H}-3, J_{3,4}=7.9\right.$ $\mathrm{Hz}), 8.59$ (d, $\left.0.8 \mathrm{H}, \mathrm{H}-6, J_{5,6}=4.21 \mathrm{~Hz}\right) .{ }^{13} \mathrm{C}$ NMR $\left(150.8 \mathrm{MHz}, \mathrm{DMSO}-\mathrm{d}_{6}\right) \delta(\mathrm{ppm}) 64.2\left(\mathrm{C}-6{ }^{\prime}\right)$, 70.1 (C-4'), 70.8 (C-2'), 71.5 (C-5'), 71.8 (C-3'), 121.6 (C-3), 126.2 (C-5), 137.8 (C-4), 149.2 (C6), $151.1(\mathrm{C}-2), 156.6(\mathrm{NC}=\mathrm{N}), 159.9\left(\mathrm{C}-1^{\prime}\right) .{ }^{1} \mathrm{H}$ NMR for $\mathbf{1 1 b} \delta(\mathrm{ppm}) 3.14\left(\mathrm{~m}, 0.2 \mathrm{H}, \mathrm{H}-5^{\prime}\right)$, 3.33 (t, $\left.0.2 \mathrm{H}, \mathrm{H}-4^{\prime}\right), 3.37$ (bd, $0.2 \mathrm{H}, \mathrm{H}-6^{\prime}, J_{6^{\prime}, 5^{\prime}}=2.9 \mathrm{~Hz}$ ), 3.48 (m, $\left.0.2 \mathrm{H}, \mathrm{H}-6^{\prime \prime}\right), 3.60$ (d, $0.2 \mathrm{H}$, $\left.\mathrm{H}-2^{\prime}, J_{1^{\prime}, 2^{\prime}}=8.6 \mathrm{~Hz}\right), 3.8\left(\mathrm{~d}, 0.2 \mathrm{H}, \mathrm{H}-3^{\prime}\right), 4.48\left(\mathrm{bs}, 0.2 \mathrm{H}, \mathrm{H}-1^{\prime}\right) .{ }^{13} \mathrm{C}$ NMR ( DMSO-d 6 ) $\delta(\mathrm{ppm})$ 61.9 (C-6'), 67.6 (C-4'), 71.3 (C-3'), 74.6 (C-2'), 78.7 (C-5'), 88.0 (C-1'); FABMS m/z = 299 $\left(\mathrm{MH}^{+\cdot}\right)$. Analylsis for $\mathrm{C}_{12} \mathrm{H}_{18} \mathrm{~N}_{4} \mathrm{O}_{5}(298.30)$ calcd: $\mathrm{C}, 48.32 ; \mathrm{H}, 6.08 ; \mathrm{N}, 18.78$; Found: C, 48.17; H, 6.01; N, $18.69 \%$.

$N^{2}$-(D-Glucose)- $N^{1}-2$-pyridylcarboxamidrazone and $N^{2}-(\alpha, \beta-D$-glucopyranosyl and $\alpha, \beta-D-$ glucofuranosyl)- $\boldsymbol{N}^{\mathbf{1}}$-2-pyridylcarboxamidrazone (12a,b,c). Hygroscopic glassy product (75 \%), ${ }^{1} \mathrm{H}$ NMR (300 MHz, DMSO-d $\left.6+\mathrm{D}_{2} \mathrm{O}\right) \delta(\mathrm{ppm}) 2.89$ (t, H-2' $\beta$ py, $\left.J_{1^{\prime}, 2^{\prime}}=7.6 \mathrm{~Hz}\right), 3.01$ (m, H-3' $\beta$ py), 3.14 (dd, H-2' $\left.\alpha, J_{1^{\prime}, 2^{\prime}}=3.6 \mathrm{~Hz}, J_{2^{\prime}, 3^{\prime}}=9.2 \mathrm{~Hz}\right), 4.28\left(\mathrm{~d}, \mathrm{H}-1^{\prime} \beta, J_{1^{\prime}, 2^{\prime}}=7.6 \mathrm{~Hz}\right), 4.90$ (d, H-1' $\left.\alpha, J_{1^{\prime}, 2^{\prime}}=3.6 \mathrm{~Hz}\right)$.

${ }^{13} \mathrm{C}$ NMR (DMSO-d 6 ) $\delta(\mathrm{ppm}) 61.7$ (C-6'), 61.5 (C-6' $\beta$ py), 70.6 (C-3' $\beta$ py), 70.9 (C-2' $\left.\alpha\right)$, 75.1 (C-2' $\beta$ py), 77.1 (C-4' $\beta$ py), 78.1 (C-5' $\beta$ py), 92.6 (C-1' $\alpha$ ), 97.2 (C-1' $\beta$ py), 162.9 (C$\left.1^{\prime}=\mathrm{N}\right)$.

\section{Acknowledgements}

The continued supports from the AvH and DFG are highly appreciated.

\section{References}

1. Neilson, D. G.; Heatlie, J. W. M.; Newlands, L. R. Chem. Rev. 1970, 70, 151.

2. (a) Mamolo, M. G.; Falagiani, V.; Vio, L.; Banfi, E. Farmaco 1999, 54, 761. (b) Mamolo, M. G.; Vio, L.; Banfi, E.; Fabris, C.; Asaro, F. Farmaco 1992, 47, 1055. (c) Banfi, E.; Mamolo, M. G.; Vio, L.; Predominato, M. J. Chemother. 1993, 5, 164. (d) Mamolo, M. G.; Vio, L.; Banfi, E.; Predominate, M.; Fabris, C.; Asaro, F. Farmaco 1993, 48, 529. (e) Mamolo, M. G.; Vio, L.; Banfi, E. Farmaco 1996, 51, 65.

3. (a) Gokhale, N.; Padhye, S.; Rathbone, D.; Billington, D.; Lowe, P.; Schwalbe, C.; Newton, C. Inorg. Chem. Commun. 2001, 4, 26. (b) Gokhale, N. H.; Padhye, S. S.; Padhye, S. B.; Anson, C. E.; Powell, A. K. Inorg. Chim. Acta 2001, 319, 90.

4. (a) Lee, K.; Hwang, S. Y.; Hong, S. W.; Hong, C. Y.; Lee, C.-S.; Shin, Y.; Kim, S.; Yun, M.; Yoo, Y. J.; Kang, M. Oh, Y. S. Bioorg. Med. Chem. 1998, 6, 869. (b) Lee, K.; Jung, W. 
H.; Park, C. W.; Park, H. D.; Lee, S. H.; Kwon, O. H. Bioorg. Med. Chem. Lett. 2002, 12, 1017.

5. (a) Lichtenthaler, F. W.; Mendel, S. Pure Appl. Chem. 1997, 69, 1853. (b) Lichtenthaler, F. W. Carbohydrates as Organic Raw Materials VCH, Weinheim: New York, 1991. (c) Cottiel, L.; Descotes, G. Treuds Heterocycl. Chem. 1991, 2, 233.

6. El Ashry, E. S. H.; El Nemer, A. Synthesis of Naturally Occurring Nitrogen Heterocycles from carbohydrates, Blackwell, 2005.

7. (a) El Ashry, E. S. H.; Awad, L. F. Carbohydr. Res. 1998, 312, 9. (b) El Ashry, E. S. H.; Awad, L. F. Nucleosides, Nucleotides \& Nucleic Acids 2001, 20, 103.

8. (a) El Ashry, E. S. H.; El Kilany, Y. Adv. Heterocycl. Chem. 1997, 67, 391. (b) El Ashry, E. S. H.; El Kilany, Y. Adv. Heterocycl. Chem. 1997, 68, 1. (c) El Ashry, E. S. H.; El Kilany, Y. Adv. Heterocycl. Chem. 1998, 69, 129.

9. El Ashry, E. S. H.; Awad, L. F.; Winkler, M. J. Chem. Soc. Perkin Trans.1 2000, 829.

10. El Ashry, E. S. H.; Abdul-Ghani, M. M. Nucleosides, Nucleotides \& Nucleic Acids 2004, 23, 567.

11. Bediak, B.; Salyan, M. E.; Pantoja, M. J. Org. Chem. 1995, 60, 8245.

12. Kubota, S.; Kirino, O.; Koida, Y.; Miyake, K. J. Pharm. Soc. Japan 1972, 92, 275.

13. (a) Khodair, A. I.; Ibrahim, E. S. I.; Diab, A. M.; Abd-El Aziz, M. M.; Omar, B. M. T.; El Ashry, E. S. H. Pharmazie 1998, 53, 294. (b) Rashed, N.; Abdel Hamid, H.; Ramadan, E.; El Ashry, E. S. H. Nucleosides \& Nucleotides 1998, 17, 1373. (c) Rashed, N.; Shoukry, M.; El Ashry, E. S. H. Bull. Chem. Soc. Jpn. 1994, 67, 149. (d) Rashed, N.; Ibrahim, E. S. I.; El Ashry, E. S. H. Carbohydr. Res. 1994, 254, 295. (e) Mousaad, A.; Rashed, N.; Ramadan, E.; El Ashry, E. S. H. Spectroscopy Lett. 1994, 27, 677. (f) Abdel-Aal, M. T.; El-Syed, W. A.; Abdel Aleem, A. H.; El Ashry, E. S. H. Pharmazie 2003, 58, 788. (g) Awad, L. F. J. Chem. Res. (S) 2001, 129. (h) Hamed, A.; Abo-Amaym, E.; El Ashry, E. S. H. Nucleosides \& Nucleotides 1998, 17, 1385.

14. (a) Simon, H.; Kraus, A. Fortschr. Chem. Fortschr. 1970, 14, 430. (b) Itano, H. A.; Matteson, J. L. Biochem. 1982, 21, 2421. (c) Itano, H. A.; Robinson, E. A. J. Am. Chem. Soc. 1961, 83, 3339. (d) Allison, W. S.; Swain, L. C.; Tracy, S. M.; Benitez, L. V. Arch. Biochem. Biophys. 1973, 155, 400. (e) Potekhim, A. A.; Zhdanov, S. I. Zh. Org. Khim. 1979, 15, 1384. (f) Takeda, Y. Carbohydr. Res. 1979, 77, 9. (g) Linek, K.; Alföldi, J.; Kucar, S.; Sticzay, T.; Novotna, Z.; Kojicproodic, B. Carbohydr. Res. 1983, 115, 259. (h) Williams, J. M. Carbohydr. Res. 1983, 117, 89. (i) Kett, W. C.; Batley, M.; Redmond, J. W. Carbohydr. Res. 1997, 299, 129. (j) Haas, J. W.; Storey, J. J. D.; Lynch, C. C. Anal. Chem. 1962, 34, 145. (k) Saeed, M.; Williams, J. Carbohydr. Res. 1980, 84, 83.

15. Petrâková, E.; Kováč, P. Carbohydr. Res. 1982, 101, 141.

16. Popsavin, V.; Grabez, S.; Stojanovic, B.; Popsavin, M.; Pejanovic, V.; Miljkovic, D. Carbhydr. Res. 1999, 321, 110. 